Check for updates

Cite this: RSC Adv., 2018, 8, 15575

\title{
Introducing copper and collagen (via poly(DOPA)) coating to activate inert ceramic scaffolds for excellent angiogenic and osteogenic capacity
}

\author{
Xu Wang, $\dagger^{\mathrm{a}}$ Chenzhou Wu, $\dagger^{\mathrm{b}}$ Hao Qi, ${ }^{\mathrm{a}}$ Meng Tian, ${ }^{\mathrm{c}}$ Huixu Xie, (D) ${ }^{\mathrm{b}}$ Yaping Wang, ${ }^{\mathrm{a}}$ \\ Zhipeng $\mathrm{Gu}^{\mathrm{d}}{ }^{\mathrm{X}} \mathrm{X}$ Peng $^{\mathrm{e}}$ and Xixiun Yu (D) *a
}

\begin{abstract}
Traditional calcium polyphosphate (CPP) scaffolds have attracted wide attention for repairing bone defects owing to their low cytotoxicity and controllable degradation. However, because of poor mechanical strength, significant brittleness and suboptimal osteoinductivity and osteoconductivity, their further clinical applications are restricted. To overcome these limitations, collagen (Col) coated $\mathrm{Cu}(I)$ ion-doped calcium polyphosphate (CCPP) scaffolds were employed and dopamine (DOPA) was used as a linkage (CCPP/D/Col) to ensure their stable and tight structure. Controllable $\mathrm{Cu}(I)$ ion continuously released from scaffolds together with collagen coating could simultaneously enhance the cytocompatibility, compressive strength and ductility, bone-related gene expression and new bone regeneration. In comparison with the initial CPP specimens, these multifunctional CCPP/D/Col composite scaffolds' crystal grains of CCPP were arranged regularly and well-ordered, and the size and rugosity were more suitable for cell spreading and attachment. Murine bone marrow stem cells (BMSCs) seeded on CCPP/D/ Col scaffolds possessed better proliferation and migration, rapid attachment and enhanced expression of osteogenic-related genes, which indicated better bone regeneration. The potential mechanism of this process was further elucidated. Both copper doping and collagen coating could effectively stabilize hypoxia-inducible factor-1 $\alpha$ (HIF-1 $\alpha)$ that thus stimulates the expression of vascular endothelial growth factor (VEGF). In addition, they could also promote the osteogenic differentiation of cells through stimulating bone-related gene expression. The concept of introducing active ions and biological macromolecules to modify inert ceramics may offer a new strategy to construct a multifunctional composite scaffold for bone tissue regeneration.
\end{abstract}

\author{
Received 6th March 2018 \\ Accepted 9th April 2018 \\ DOI: $10.1039 / \mathrm{c} 8 \mathrm{ra01960f}$ \\ rsc.li/rsc-advances
}

\section{Introduction}

Bone tissue regeneration is a complex biological process, in which the proliferation and differentiation of target cells are guided by some released specific factors, the guided growth of bone is activated and accompanied by microvessel formation., ${ }^{\mathbf{1 , 2}}$ An ideal bone repair material for regeneration of bone defects should possess high osteoconductivity, good mechanical strength and the ability to effectively induce osteogenesis and angiogenesis for accelerating vascularization, which will further

${ }^{a}$ College of Polymer Science and Engineering, Sichuan University, Chengdu 610065, China.E-mail: yuxixun@163.com

${ }^{b}$ Department of Head and Neck Oncology, West China Hospital of Sichuan University, Chengdu, Sichuan 610021, P. R. China

${ }^{c}$ Department of Neurosurgery, West China Hospital of Sichuan University, Chengdu, Sichuan 610041, P. R. China

${ }^{d}$ Guangdong Provincial Key Laboratory of Sensor Technology and Biomedical Instrument, School of Engineering, Sun Yat-sen University, Guangzhou, 510275, P. R. China

${ }^{e}$ Experimental Animal Center of Sichuan University, Chengdu 610065, P. R. China

$\uparrow$ These authors contributed equally. promote new bone formation., ${ }^{3,4}$ Moreover, biomaterials with porous structures, which benefit cell spreading and attachment, are also important. ${ }^{5}$ Calcium polyphosphate (CPP) was regarded as a novel bone substitute material with a similar element composition to that of natural bones. In the last 10 years, CPP as well as congeneric bioceramics with controllable degradation and good bioactivity have been extensively applied for bone regeneration. However, its angiogenesis and osteogenesis properties seemed to be insufficient. Meanwhile, similar to normal inorganic ceramics, CPP's further clinical application for weight-bearing bone reconstruction was restricted by its brittleness and low compressive strength. ${ }^{6,7}$ Thus, the combination of inherent bioactivity and controllable degradation of CPP scaffolds with additional optimal ductility, excellent angiogenesis and osteogenesis property is desired. If realized, these multifunctional scaffolds would be very useful for overcoming the major challenges faced by current bone repair scaffolds.

Recently, incorporating bioactive ions or biogenic macromolecules into inert scaffolds to stimulate angiogenesis or osteogenesis differentiation of cells for bone reconstruction has 
become of interest. ${ }^{8-10}$ As a bioactive inorganic ion, divalent copper ion $\left(\mathrm{Cu}^{2+}\right)$ doped into bioceramics plays an important role in blood vessel regeneration as a trace element. It has been proved that the incorporation of $\mathrm{Cu}^{2+}$ in moderation could effectively promote angiogenesis through artificially mimicking hypoxia to stabilize the expression of HIF- $1 \alpha$ and further stimulate VEGF expression. ${ }^{\mathbf{1 1} 12}$ In consideration of the vital functions it exerts in the processes of cell differentiation and blood vessel formation, it may thus provide a channel in bone reconstruction and regeneration through stimulating the expression of bone-related genes. ${ }^{\mathbf{1 3 - 1 5}}$

Meanwhile, as a major component of the extracellular matrix, collagen was selected as an ideal biomacromolecule for secondary modification of the CPP scaffolds owing to its critical role in tissue formation and stimulating the secretion of some cell growth factors. ${ }^{\mathbf{1 6 - 1 8}}$ Moreover, the excellent bioactivity and biocompatibility could accelerate the formation of a bone-like mineralization layer on its surface. ${ }^{19}$ Therefore, it would be a good choice to chemically modify porous inorganic scaffolds with collagen. Our previous work combined a bioceramic scaffold with collagen via glutaraldehyde (GA) crosslinking; however, its physical encapsulation usually suffered from serious phase separation. ${ }^{\mathbf{1 0}}$ In recent years, dopamine (DOPA) has attracted increasing attention as a bio-adhesive for its easy solvent-free operation, non-toxicity and excellent adherent properties for most materials. ${ }^{20-22}$ DOPA is easy to selfpolymerize, forming poly(DOPA), which possesses a large amount of active functional groups and can react with most materials. Thus, it might be an effective approach to modify inert CPP ceramics via coating the collagen layer with DOPA, which could stabilize their connection and promote their phase compatibility. Nevertheless, the synergetic biological effects of $\mathrm{Cu}^{2+}$ and collagen released from composite scaffolds on cell responses have been ignored until now. Considering the characteristics of $\mathrm{Cu}^{2+}$ and the advantages exclusively exhibited by collagen, it was feasible to develop innovative biocompatible and bioresorbable composite scaffolds with excellent angiogenesis and osteogenesis properties through introducing $\mathrm{Cu}^{2+}$ ions and collagen. Combining them with inert CPP scaffolds to acquire multifunctional organic/inorganic composite scaffolds and improve its effect on bone restoration is promising. ${ }^{23}$ It can be inferred that collagen's modification endowed inorganic scaffolds with eliminated brittleness, controllable releasing bioactive ions and excellent cytocompatibility, which may prevent additional surgical interventions for patients. In addition, to more accurately model the osteogenic differentiation promoted by the tested materials in vivo, murine bone marrow stromal cells (BMSCs) are generally regarded as the most important seed cells in bone tissue engineering. ${ }^{24,25}$ Thus, the interaction of BMSCs and scaffolds was emphatically discussed to evaluate the osteogenic tendency obtained with the modified scaffolds.

Herein, we firstly doped a series of concentrations of copper ions inside the scaffolds, and among them, selected a moderate doping concentration through CCK-8 assay and mechanical measurement. Secondary modification was carried out via coating the collagen layer with DOPA, hoping to form a stable connection and preventing phase separation. The biocompatibility and angiogenesis and osteogenesis properties of this scaffold, which are tightly connected to bone reconstruction, were systematically researched. Through preparing CCPP/D/Col composite scaffolds, we hope to endow traditional inert bioceramics with osteoconductivity, sufficient toughness, and effective osteogenesis and angiogenesis inducing capability to accelerate vascularization and further promote new bone formation. It would provide a promising strategy to combine bioactive copper ions and collagen with inert CPP scaffolds to construct multifunctional scaffolds for bone regeneration.

\section{Materials and method}

\subsection{Scaffolds fabrication and characterization}

Porous CPP scaffolds containing a series of concentrations of copper ranging from copper-free to $0.5 \%$ (mole fraction, incorporating $0 \%, 0.01 \%, 0.05 \%, 0.2 \%$ and $0.5 \%)$. These scaffolds were synthesized as previously reported. Briefly, $\mathrm{CaCO}_{3}$ and $\mathrm{Cu}_{2}(\mathrm{OH})_{2} \mathrm{CO}_{3}$ were slowly added into $85 \%$ phosphoric acid with constant stirring. After reacted at $25{ }^{\circ} \mathrm{C}$ for $12 \mathrm{~h}$, products were obtained through evaporating under vacuum and the precipitates were washed with ethanol several times until the $\mathrm{pH}$ was about 7. Subsequently, these homogeneous amorphous CCPP were sintered at $800{ }^{\circ} \mathrm{C}$ for $10 \mathrm{~h}$ to polymerize and then heated to $1100{ }^{\circ} \mathrm{C}$ with a $15{ }^{\circ} \mathrm{C} \mathrm{min}^{-1}$ heating rate to melt. The melt was rapidly quenched in distilled water to cool down, then amorphous frits were milled and screened to yield powders in a size range of $<75 \mu \mathrm{m}$. Finally, CCPP scaffolds were prepared through mixing the powers with stearic acid and $3 \mathrm{wt} \%$ poly(vinyl alcohol), pressed at $98 \mathrm{MPa}$, and then sintered again to form a porous structure. Since $\mathrm{Cu}^{2+}$ could react with phosphate more rapidly than $\mathrm{Ca}^{2+}, \mathrm{Cu}_{2}(\mathrm{OH})_{2} \mathrm{CO}_{3}$ (Kelong Chemical Reagent Company, China) was added to replace an equal amount of $\mathrm{CaCO}_{3}$ during the solution preparation process and the corresponding CCPP scaffolds were obtained (the porosity of all the scaffolds was about $65 \%) .{ }^{10}$ Then, CCPP and/or CPP were immersed in a $2 \mathrm{mg} \mathrm{ml}^{-1}$ DOPA (Sigma Company, USA) solution (10 mM Tris-HCl buffer solution as solvent, $\mathrm{pH}$ 8.5) to coat DOPA, forming D-CCPP or D-CPP scaffolds. ${ }^{26,27}$ Subsequently, the CCPP/D/Col scaffolds were prepared as follows: a $5 \mathrm{mg} \mathrm{ml}{ }^{-1}$ collagen solution was prepared in $0.5 \mathrm{M}$ acetic acid $(\mathrm{pH}=3-4)$, in which D-CCPP scaffolds were immersed. At room temperature, $0.2 \mathrm{ml}$ of $\mathrm{NaIO}_{4}$ solution $(0.01 \mathrm{M})$ was added dropwise for oxidative induction in further modification to obtain the ultimate CCPP/D/Col composite scaffolds. After drying under vacuum conditions, the resultant modified scaffolds were characterized.

To authenticate the homogeneity of the CPP or CCPP powders, the amorphous frits were milled and screened, and their particle sizes and particle size distributions were determined using a Mastersizer 2000 laser particle size analyser before being pressed into round pieces. After that, scanning electron microscope (SEM) examination was employed to observe the surface topography, phase constitution and porous microstructure of the prepared scaffolds. For the latter process, changes in the chemical composition resulting from the 
modification of the self-polymerized DOPA film and the exterior immobilized collagen on the coating were determined using Fourier transform infrared (FTIR) spectroscopy (Nicolet 560, USA).

\subsection{Compressive strength assessment and fracture surface observation}

The compressive strength of these cylindrical scaffolds, with dimensions of $10 \mathrm{~mm}$ in diameter and $10 \mathrm{~mm}$ in thickness, was measured by electronic mechanical testing machine (Instron 4302 , USA). A loading rate of $0.5 \mathrm{~mm} \mathrm{~min}^{-1}$ was applied to reach a fracture point as reported by Hayashi in the same measurement of hydroxyapatite. ${ }^{28}$ During the process of compression treatment, once a sample had fractured, a dramatic decrease occurred in the force applied to the specimens. The first maximum load was recorded and performed as the sample's compressive strength. In each group, at least five specimens were tested and the results are expressed as average \pm standard deviation (SD).

In addition, to evaluate the ductility of these compound scaffolds, their fracture surface morphologies were observed by SEM at $20 \mathrm{kV}$. After being frozen in liquid nitrogen for at least $5 \mathrm{~min}$, the samples were fractured immediately. Then, the fracture surface was coated with gold and observed.

\subsection{Release of copper and calcium ions from compound scaffolds}

As previously reported, $\mathrm{Cu}^{2+}$ or the synergism of $\mathrm{Cu}^{2+} / \mathrm{Ca}^{2+}$ exhibited significant promotion of vascularization, which may further accelerate the formation of new bones. ${ }^{29,30}$ To investigate their ion release rates, the CCPP or CCPP/D/Col scaffolds were immersed in simulated body fluid ( $\mathrm{SBF}, \mathrm{pH}=7.4$ ) at $37^{\circ} \mathrm{C}$ for various periods up to $30 \mathrm{~d}(3 \mathrm{~d}, 6 \mathrm{~d}, 12 \mathrm{~d}, 18 \mathrm{~d}, 21 \mathrm{~d}, 27 \mathrm{~d}$ and 30 d). After incubation treatment at each designated time point, the scaffolds were withdrawn and their leaching liquids were collected to measure the release rates and cumulative release amounts of copper and calcium ions. The solution was refreshed daily and analyzed by inductive coupled plasma atomic emission spectrometry.

\subsection{Cell culture and proliferation rate assay}

Murine bone marrow stromal cells (BMSCs) isolated from a 2 weeks-old SD rat were employed to evaluate the effect of $\mathrm{Cu}^{2+}$ incorporation and collagen coating on cell proliferation, attachment, migration and differentiation. After cutting off both ends of the epiphysis, bone marrow inside the rat's femurs was rinsed out using DMEM culture medium. Following centrifugation and disassociation treatment, the obtained cells were moved into low-glucose Dulbecco's modified Eagle's medium (L-DMEM, Hyclone) supplemented with $10 \%$ fetal bovine serum (FBS, Gibco, USA) and cultured in an incubator with humidified atmosphere $\left(37{ }^{\circ} \mathrm{C}, 5 \% \mathrm{CO}_{2}\right){ }^{31}$ The culture medium was refreshed every two days to remove any suspended cells (especially haemocytes) for purification. Meanwhile, all samples were lyophilized and sterilized using $\gamma$-irradiation at 25 kGy in advance.
For the cell proliferation assay, after adding $500 \mu \mathrm{L}$ of complete medium to moisten the scaffolds for at least $4 \mathrm{~h}$, the medium was wiped off and BMSCs at passage 3 were seeded on them at a density of $2 \times 10^{4}$ cells per well, continuously cultivating for predetermined periods $(1 \mathrm{~d}, 3 \mathrm{~d}, 5 \mathrm{~d}$ and $7 \mathrm{~d})$. At each time point, $50 \mu \mathrm{L}$ of CCK-8 solution was added to each construct and then incubated at $37^{\circ} \mathrm{C}$ for $2 \mathrm{~h}$. Subsequently, an aliquot of $150 \mu \mathrm{L}$ was taken from this mixed solution and transferred to a new 96-well plate for optical density (OD) measurement. The absorbance was recorded at $\lambda=405 \mathrm{~nm}$ with a Microplate Reader (Model550, Bio Rad Corp.) and BMSCs seeded on fresh culture medium were used as the control.

\subsection{Cell attachment morphology and distribution}

After the BMSCs had been seeded on sterilized scaffolds at a density of $5 \times 10^{4}$ cells per $\mathrm{ml}$ and incubated for 2 days, the cellular morphology was observed by scanning electron microscopy (SEM). In detail, cells were rinsed with PBS several times to remove cellular secretions and then scaffolds with attached cells were fixed with $3 \%$ GA for $12 \mathrm{~h}$ at $4{ }^{\circ} \mathrm{C}$. Subsequently the samples were dehydrated with gradient concentrations of ethanol $(25 \%, 50 \%, 70 \%, 80 \%, 90 \%, 95 \%, 98 \%$ and $100 \%$ ), and then critical point-dried before SEM (JSM-5900LV, JEOL, Japan) experiments.

To evaluate the cytoskeletal organization on or across the composite scaffolds, BMSCs were stained with DAPI/rhodamine phalloidin and observed by confocal laser scanning microscope (CLSM). After 4 days of incubation using the same method as for the SEM experiment, cells attached on scaffolds were rinsed with PBS solution three times and fixed with $4 \%$ paraformaldehyde for $10 \mathrm{~min}$ at room temperature. Subsequently, they were further treated in $0.5 \%$ Triton $\mathrm{X}-100$ for $5 \mathrm{~min}$ to permeabilize the cell membranes, followed by a brief rinse with PBS, then a fluorescein stain using $10 \mathrm{mM}$ rhodamine phalloidin solution was employed to reveal cytoplasmic visualization, while the cell nuclei were counterstained using $5 \mu \mathrm{g} \mathrm{ml}^{-1}$ DAPI solution. Finally, cytoskeletal organization on various scaffolds was visualized by CLSM.

\subsection{Alizarin red-S (ARS) staining}

To evaluate their osteogenic properties, cell-mediated matrix mineralization tightly adjacent to various scaffolds was qualitatively detected using ARS assay. Alizarin red could sensitively react with the calcium deposits generated from osteoblastic differentiation and form red plaque.

In detail, after seeding BMSCs on or tightly appressed to disks and culturing for 21 days in sufficient medium, the scaffolds/cells were simultaneously fixed in $4 \%$ paraformaldehyde for $1 \mathrm{~h}$, rinsed with PBS and then stained by $1 \%$ Alizarin red solution $(\mathrm{pH} \approx 4.2)$ for 30 min with constant shaking. To reduce deviation, there were two replicate samples per group. Subsequently, the stain was repeatedly rinsed with deionized water to remove residual ARS, and then air-dried and imaged using a camera attached to an inverted microscope. 


\subsection{Real-time quantitative polymerase chain reaction (RT- qPCR)}

With regard to modification of traditional CPP scaffolds, the effects of copper doping and collagen coating on osteogenic differentiation and angiogenic properties were determined through RT-qPCR to measure the mRNA expression of osteogenesis-(ALP, OCN, OPN) and angiogenesis-related genes (HIF$1 \alpha$, VEGF). Briefly, BMSCs were seeded on three identical specimens in each group at a density of $1 \times 10^{5}$ cells per $\mathrm{ml}$ and incubated for 21 days. Subsequently, to extract total RNA, TRIzol reagent (Invitrogen, USA) was added and harvested cells with entirely isolated from RNase. The complementary DNA was reverse-transcribed from total RNA using a PrimeScript RT reagent Kit (TaKaRa ${ }^{\circledR}$, China) according to the specifications for RT-qPCR, and various RT-qPCR primers were designed based on cDNA sequences from the NCBI sequence database. Afterwards, RT-qPCR was performed on a real-time fluorescence quantitative instrument (PIKORed 96, Thermo Fisher Co., Ltd, USA) using SYBR Premix Ex Taq II Kit reagent (TaKaRa®, China). The relative expressions of these target genes were detected through normalizing the gene of interest to the reference gene $\beta$-actin, and it was calculated using the following formula:

Relative expression $=2^{- \text {(normalized average Cts })} \times 10^{4}$

\subsection{In vivo animal study}

All experimental animals were cared for according to the guidelines formulated by the National Institutes of Health on the humane use and care of laboratory animals, and all procedures performed on animals were approved by the laboratory animal center of Sichuan University. In this experiment, a total of $35 \mathrm{SD}$ rats (3 months old, 250-300 g) were randomly divided into five groups. Based on the results obtained in cell culture experiments and gene expression measurements, the CCPP scaffolds doped with $0.05 \%$ copper ions (0.05\% CCPP) and products secondarily modified by collagen $(0.05 \% \mathrm{CCPP} / \mathrm{D} / \mathrm{Col})$ were selected for further intravital evaluation. Meanwhile, the undoped CPP scaffolds and commonly used HA scaffolds served as the controls.

After the rats were anaesthetized through intraperitoneally injecting pentobarbital at a concentration of $40 \mathrm{mg} \mathrm{kg}^{-1}$ under aseptic conditions, a 1.0-1.2 cm longitudinal incision was made on their scalp, the periosteum on the bone surface was separated and removed, and then the calvarium was exposed through blunt separation. Subsequently, a full thickness defect $(\Phi=10 \mathrm{~mm})$ was created in the center area of the calvaria by using a bone drill. Meanwhile, they were constantly irrigated with sterilized $0.9 \%$ saline to avoid local overheating and tissue necrosis. After that, CPP, 0.05\% CCPP, 0.05\% CCPP/D/Col and HA scaffolds with dimensions of $\Phi 10 \times 2 \mathrm{~mm}$ were carefully implanted into defects. Following surgery, peripheral soft tissues were sutured layer by layer using a 4-0 surgical suture. To prevent wound infection, all animals received a daily injection of penicillin for 5 days post-surgery. After 8 weeks post-surgery, the rats were euthanized. Within the shortest time, implanted scaffolds with surrounding bone tissues were harvested and fixed in $4 \%$ paraformaldehyde for $48 \mathrm{~h}$ preparing for micro-CT scanning. The defects were observed at a resolution of $18 \mu \mathrm{m}$ and $3 \mathrm{D}$ images of bone formation were obtained.

\section{Results and discussion}

\subsection{Porous scaffold fabrication and characterization}

In design, a fraction of $\mathrm{Ca}$ ions in the initial CPP scaffolds was easily replaced by $\mathrm{Cu}^{2+}$ since copper had a critical application to improve the angiogenic and osteogenic capacity. As seen from the arrangement of extra nuclear electrons, $\mathrm{Cu}$ possessed a lone pair electron in the $4 \mathrm{~s}$ orbit and it had a stronger coordination tendency with $\mathrm{O}$, which was probably the reason why $\mathrm{Cu}^{2+}$ could react with the ligand (phosphate) rapidly and form a more stable complex compared with $\mathrm{Ca}^{2+}$. During the degradation of $\mathrm{CCPP}, \mathrm{Ca}^{2+}$ and $\mathrm{Cu}^{2+}$ were continuously released for breakage of $\mathrm{P}-\mathrm{O}$ bonds, and then exhibited synergistic promotion of osteogenesis and vascularization.

The typical morphologies of the scaffolds modified by different methods are shown in Fig. 1(A). As exhibited, doping the initial CPP ceramics with $0.01-0.5 \% \mathrm{Cu}$ had little effect on the microstructure of the scaffolds: the open interconnected porosity which satisfied the requirements of cell growth and nutrient/metabolic waste transportation was well-preserved and the circular apertures were still distributed evenly (their pore sizes all ranged from $100 \mu \mathrm{m}$ to $400 \mu \mathrm{m}$ and the porosity was maintained at about $65 \mathrm{vol} \%$ as well). The grains of CCPP were arranged regularly and they were connected with each other more intimately, forming a more compact surface structure and bulk. This may be attributed to $\mathrm{Cu}^{2+}$ ions having an agglutination effect from being surrounded by two non-bridging oxygen atoms, and compared to the $\mathrm{Ca}-\mathrm{O}$ bond, the $\mathrm{Cu}-\mathrm{O}$ bond revealed more covalent character. Coating DOPA onto CCPP scaffolds had little effect on the microstructure and surface topography: it was hard to distinguish CCPP and D/CCPP samples through differences in pore size and number.

To confirm that the DOPA molecule was successfully introduced, random regions on scaffolds were analyzed using a scanning electron microscope equipped with energydispersive spectrometer (SEM-EDS). As revealed, a new peak representing nitrogen element appeared in the EDS spectrum of the D/CCPP sample. It indicated that the inorganic scaffold was successfully modified by DOPA and their chemical reaction formed a great amount of stable bridge bonds for connection. The poly(DOPA) layer on inorganic scaffold could effectively activate the inert CCPP scaffold and concatenate multiple functional groups on the surface; however, it may be problematic for cell attachment and migration. ${ }^{32}$ Therefore, for the ultimate coverage of the collagen coating, its secondary modification was necessary to promote biocompatibility. Covering with collagen layers resulted in an apparent change in the particulate and porous microstructure to a much denser and smoother surface (although the scaffolds' porous surface was completely covered by collagen to form a smooth surface, the 
(A)
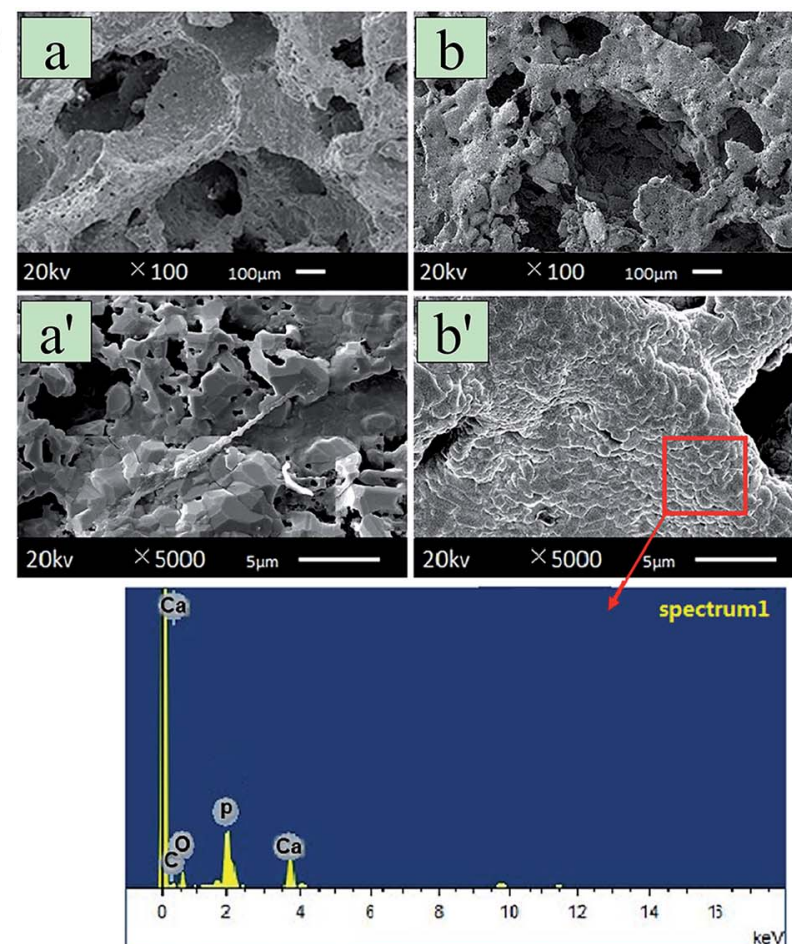
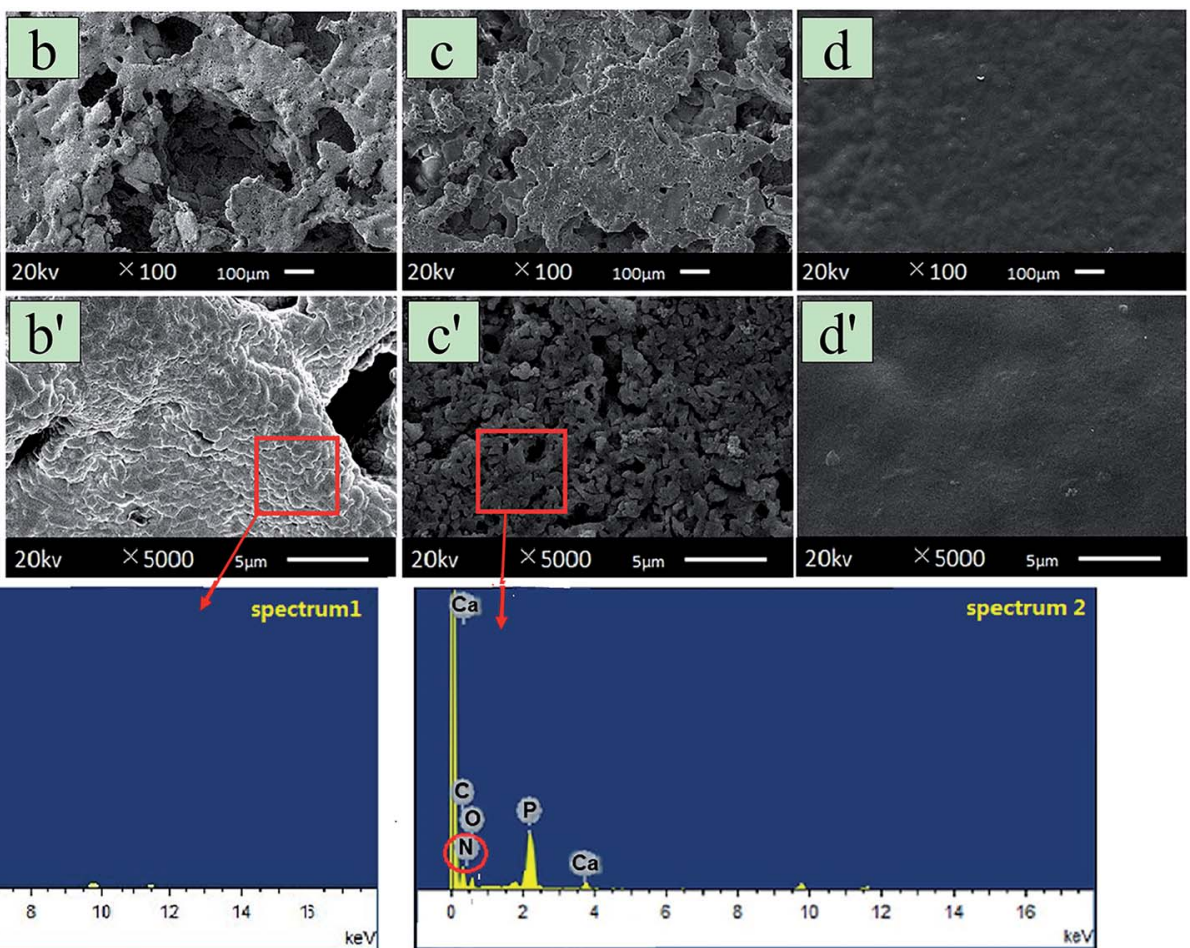

(B)

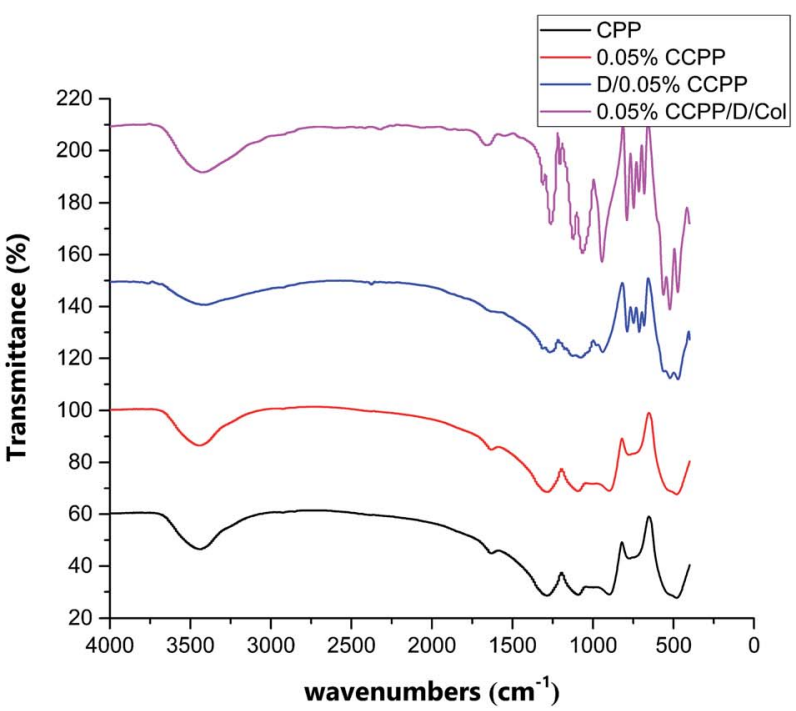

Fig. 1 (A) The typical morphologies of scaffolds modified by different methods: (a and $\left.a^{\prime}\right)$ CPP, (b and b') 0.05\% CCPP, (c and c') D/0.05\% CCPP, ( $d$ and $d^{\prime}$ ) D/0.05\% CCPP/Col and confirmatory SEM-EDS analysis for 0.05\% CCPP and D/0.05\% CCPP specimens to certify DOPA modification. (B) FTIR spectra of various CCPP scaffolds before and after modification.

collagen layer was easy to degrade and was then dedicated to promoting cell attachment and migration).

In Fig. 1(B), FTIR spectroscopy was applied to analyze the chemical structures of these unmodified or modified scaffolds. There were no marked differences in the spectra for CPP and $0.05 \%$ CCPP, which means that $\mathrm{Cu}$ could be doped without creating new peaks in the FTIR spectra. In the spectra for the D/ CCPP sample spectra, resonances appearing at $787.4 \mathrm{~cm}^{-1}$ and $748.3 \mathrm{~cm}^{-1}$ were attributed to substitution happening at the 1 , 2 , and 4 positions of the benzene ring, which further corroborated that the CCPP scaffolds were successfully modified by the poly(DOPA) layer. Subsequent to collagen coating, the absorption bands corresponding to amido linkage were enhanced markedly and dominated the spectrum: peaks centered at $3428.6 \mathrm{~cm}^{-1}$ and $3082.0 \mathrm{~cm}^{-1}$ were attributed to the stretching vibrations of $\mathrm{N}-\mathrm{H}$ groups for amide $\mathrm{A}$ and $\mathrm{B}$ bands. The amide I presented at $1661.2 \mathrm{~cm}^{-1}$ and amide II at $1542.8 \mathrm{~cm}^{-1}$ were mainly associated with $\mathrm{C}=\mathrm{O}$ resonance vibrations in peptide and $\mathrm{N}-\mathrm{H}$ bending vibrations coupled to C-N stretching vibration, respectively. In addition, amide III at 
$1260.3 \mathrm{~cm}^{-1}$ was caused by $\mathrm{C}-\mathrm{N}$ bending stretching, overlapped with $\mathrm{CH}_{2}$ groups' wagging vibration. Since collagen was the only compound in the modified scaffolds that could introduce an amido bond, this result indicated that collagen was closely coated onto the surface of the scaffolds and formed a stable chemical structure.

\subsection{Compressive strength assessment and fracture surface observation}

For clinical application, bone repair scaffolds should have sufficient mechanical strength for bone regeneration at the site of fractures or defects and maintain their reticular microstructure intact, which may be beneficial to cell growth. Therefore, compressive strength assessment and fracture surface observation were conducted to characterize the effect of $\mathrm{Cu}$ doping and/or collagen incorporation on the mechanical strength and ductility compared to the initial inorganic scaffolds. ${ }^{33}$

As Fig. 2(A) reveals, even though the mechanical strength of the CPP scaffold was significantly higher than that of traditional HA (it has gained wide clinical application and its strength was $0.38 \mathrm{MPa}$ ), it still hard to reach international requirements for application as repair materials to replace defective cancellous bone and need further development. ${ }^{32}$ The compressive strength of these CPP composite scaffolds could be varied by changing the concentration of copper doped or by further modification by collagen. As exhibited, the strength is only 2.8 MPa for unmodified CPP, while increasing the $\mathrm{Cu}$ content increased the strength. At $0.05 \%$ CCPP the strength had nearly doubled to $5 \mathrm{MPa}$ and approached the value for cancellous bone. Although continuously doping $\mathrm{Cu}$ could further increase the compressive strength, serious cytotoxicity limited their application. Therefore, combined with the tests below about biological responses, $0.05 \%$ CCPP was selected as the optimal scaffold material for suitable copper release rate and improved mechanical properties. The increased compressive strength with increasing $\mathrm{Cu}^{2+}$ concentration may be attributed to the following reasons: $\mathrm{Cu}^{2+}$ had a stronger electrostatic attraction for anions than $\mathrm{Ca}^{2+}$ and it was easy to displace the position of $\mathrm{Ca}^{2+}$, increase the attraction for cations and promote the formation of regular crystals. $\mathrm{Cu}^{2+}$ functioned as a covalentcharacterized adhesive in porous scaffolds and these newly formed tiny crystals tended to "agglutinate" more tightly.

The $\mathrm{Cu}$ doping improved the compressive strength of the initial inorganic scaffold and collagen incorporation further eliminated its brittleness. ${ }^{34}$ After secondary modification by DOPA-Col coating, the compressive strength of these composite scaffolds was dramatically enhanced. Especially, Col coating increased the compressive strength of $0.05 \%$ CCPP from 4.7 MPa to 5.3 MPa. This may be because DOPA introduction effectively guaranteed a tight connection between the inorganic and organic phases and collagen itself possesses excellent ductility. ${ }^{35}$

The fracture surface morphology of the $\mathrm{D} / \mathrm{CCPP} / \mathrm{Col}$ group is exhibited in Fig. 2(B); most of the spaces between the CCPP particles were filled by adherent collagen. The laminated collagen coating was adhered tightly and distributed evenly on the surface of the CCPP scaffolds, and no conspicuous aggregations appeared in sight. Under larger magnification $(\times 5000)$, some of the collagen was embedded in the pore walls and some others were connected through pores forming a threedimensional network microstructure. Since the excellent ductility possessed by collagen itself and this kind of structure could effectively absorb fracture energy and prevent propagation of cracks, DOPA-Col modification endowed CCPP scaffolds with greater compressive strength and better ductility. ${ }^{36}$ It could prevent small cracks diffusing in the CCPP phase and enabled the scaffolds to resist multiple cracking events before totally collapsed. On the contrary, once small cracks appeared on the CCPP scaffold, they propagated rapidly without prevention. More than that, once a lesser force was imposed, the HA sample collapsed immediately before cracks formed and exhibited
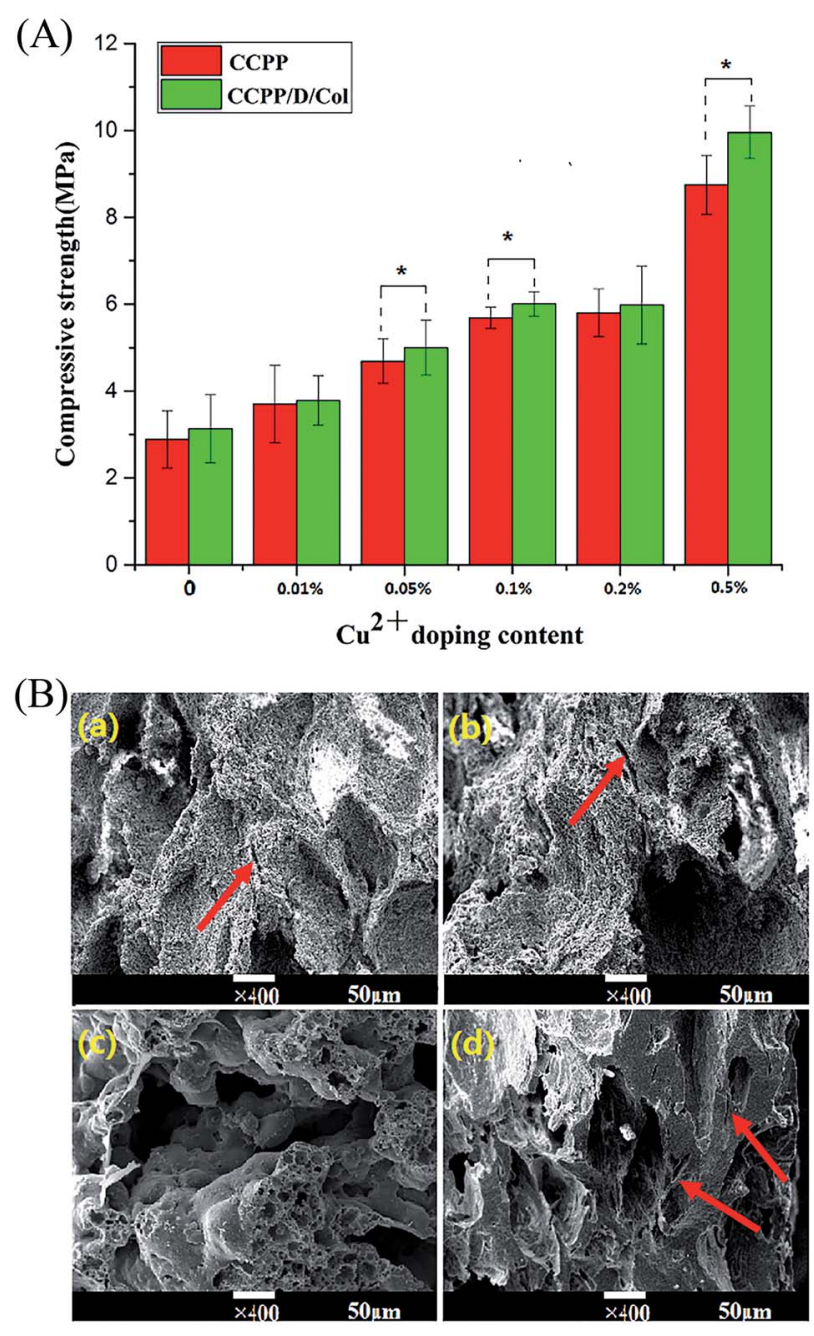

Fig. 2 (A) Compressive strength of 0.05\% CCPP and 0.05\% CCPP/D/ Col composite scaffolds. The addition of copper doping and collagen coating increased the compressive strength of the scaffolds. For DOPA/Col coated CCPP scaffolds, * means a significant difference compared with the corresponding CCPP scaffolds before modification, $* p<0.05$. (B) Cross section SEM images of various scaffolds. (a) $0.05 \%$ CCPP, (b) D/0.05\% CCPP, (c) $0.05 \%$ CCPP/D/Col, and (d) hydroxyapatite (HA). Red arrow: small cracks diffusing on scaffolds. 
more palpable brittleness. It indicated that, superior to doping $\mathrm{Cu}$ inside CPP scaffolds, being further modified by collagen could more effectively increase the scaffolds' compressive strength and eliminate frangibility.

\subsection{Ion release of $\mathrm{CCPP}$ and/or $\mathrm{CCPP} / \mathrm{D} / \mathrm{Col}$ scaffolds in SBF}

Even though a low concentration of $\mathrm{Cu}^{2+}$ could effectively promote vascularization, high levels of it may cause significant cytotoxicity and serious pain stimulation. ${ }^{29}$ In this research, it was controlled through only loading limited amounts of $\mathrm{Cu}$ inside and fabricating relatively stable bonding connections, making the bonds break successively and maintain for a longer period. During the process, the ion release rate was measured in real-time using ICP-AES. As Fig. 3 reveals, for various CCPP groups, the cumulative released amounts of $\mathrm{Cu}^{2+}$ and $\mathrm{Ca}^{2+}$ showed a conspicuous dependence on the initial $\mathrm{Cu}$ concentration doped inside the scaffolds: as copper doping increased, a significant increase in $\mathrm{Cu}^{2+}$ and $\mathrm{Ca}^{2+}$ release was observed. Once the concentration of copper doped exceeded $0.1 \%$, the releasing amount of $\mathrm{Cu}^{2+}$ and $\mathrm{Ca}^{2+}$ increased dramatically. The
$0.2 \%$ and/or $0.5 \%$ CCPP scaffolds possessed the highest $\mathrm{Cu}^{2+}$ release rates and total release amounts (their cumulative release amounts reached $0.0053 \mathrm{mg}$ and $0.0058 \mathrm{mg}$, respectively), which may cause serious cytotoxicity. The amount of $\mathrm{Cu}^{2+}$ released from $0.05 \%$ CCPP scaffolds was considerably lower: it kept a nearly constant release rate and the maximum release amount was only $0.0046 \mathrm{mg}$, which was sufficient to stimulate new bone regeneration and induce guidance of vascularization. Therefore, $0.05 \%$ CCPP was selected for use in further research owing to its excellent biocompatibility.

A similar release tendency was obtained in CCPP/Col compound group. However, for CCPP and CCPP/Col samples doped with equivalent amounts of copper, the CCPP samples released more rapidly and continued for a shorter period. For the CCPP groups, without a burst release phenomenon at the initial stage, they maintained a constant release rate for subsequent days and reached a peak value on the $27^{\text {th }}$ day, whereas the release rate in the equivalent CCPP/Col group slowed considerably and its cumulative release amount of $\mathrm{Cu}^{2+}$ was reduced by at least $0.001 \mathrm{mg}$. Therefore, the release rate and cumulative content of $\mathrm{Cu}^{2+}$ and $\mathrm{Ca}^{2+}$ in aggregate not only

\section{(a)}
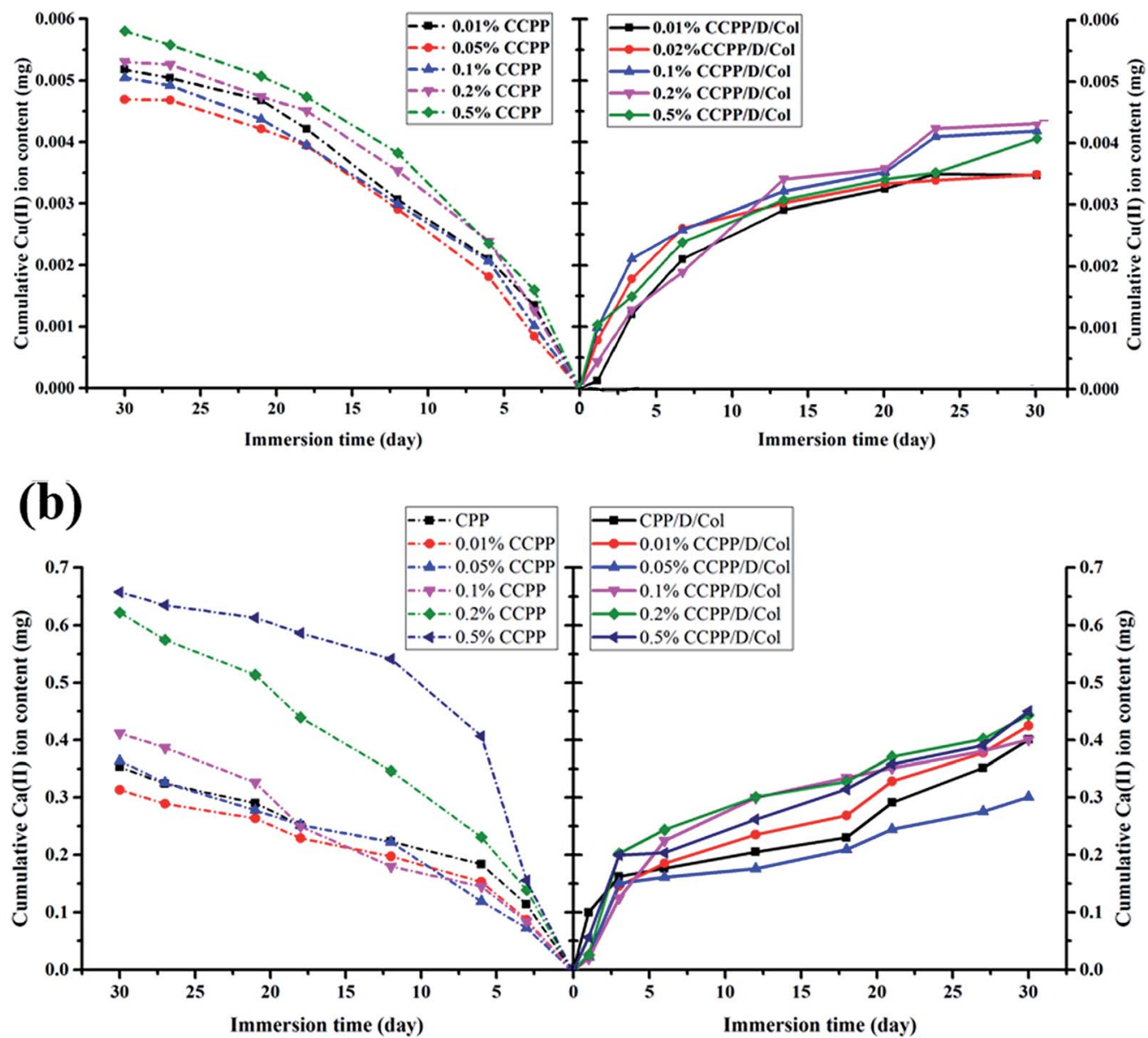

Fig. 3 ICP-OES analysis: cumulative amount of (a) $\mathrm{Cu}^{2+}$ ions and (b) $\mathrm{Ca}^{2+}$ ions released from various scaffolds before and after collagen modification. 
depended on the amount of $\mathrm{Cu}$ incorporation inside initial CPP scaffolds but also on further modified by collagen. It may be attributed to following reasons: in the first step, the DOPA/Col coating could form an effective physical barrier on surface, preventing the CCPP scaffolds from rapid degradation and dissolution. Then, the ion infiltration and diffusion transferred from the unmodified CCPP scaffolds turned the proximal microenvironment to a lower $\mathrm{pH}$ and these acidic conditions may accelerate the degradation process and ion release. Nevertheless, scaffolds further modified by DOPA/Col had the ability to automatically adjust or retain the $\mathrm{pH}$ value at a neutral level, which could effectively delay the degradation process and ion release. Above all, collagen contains a peptide sequence structure similar to that of RGD tripeptide, which has been widely employed for surface medication to promote cell proliferation, adhesion, migration and differentiation. ${ }^{29}$ Therefore, this secondary modification could further promote its biocompatibility and make it possess better angiogenic and osteogenic properties.

\subsection{Cell proliferation}

The CCK-8 assay was performed to compare the proliferation rate of BMSCs cultured on CPP scaffolds doped with different concentrations of $\mathrm{Cu}^{2+}$ and/or introduced $\mathrm{Col}$ modification. As shown in Fig. 4, a significantly increasing trend of cell growth was observed with time. Briefly, cell numbers in every group remained at the same level for the initial 3 days and began to exhibit a dramatically higher proliferation rate from the 5 th day.

According to previously established procedures, $\mathrm{Cu}$ (II), as one of the most important trace element in vivo, has excellent biocompatibility at low concentrations. ${ }^{14,37}$ It could effectively promote vascularization and may be closely linked with osteogenesis. Some results in this research demonstrated that an appropriate amount of $\mathrm{Cu}^{2+}$ introduction exhibited a deferred positive effect on BMSC proliferation: comparing their OD value exhibited at the same time point, with increased $\mathrm{Cu}^{2+}$ concentration, the cell numbers cultured on corresponding scaffolds increased simultaneously. The peak value was reached at a certain concentration of $0.05 \%$. As the $\mathrm{Cu}^{2+}$ concentration further increased, cell numbers began to decrease since high concentrations of $\mathrm{Cu}^{2+}$ exhibit high cytotoxicity, which may inhibit cell growth. Therefore, $0.05 \%$ CCPP was selected as the optimal scaffold for better cytocompatibility and attractive potential for vascularization.

Besides, as can be seen from Fig. 4, when collagen was present on the surface of the inorganic scaffolds, cellular proliferation took place rapidly. A slight advantage in the OD values of collagen coated groups was exhibited from the $1^{\text {st }}$ day, which can only be attributed to the promoting effect of collagen for cell attachment. Superior to various unmodified CCPP scaffolds, a significantly higher cell proliferation of BMSCs was observed, attributed to the collagen coating, after 3 days' culture. On the last day (7th day), compared with the corresponding CCPP group, the composite scaffolds with collagen coating exhibited an increase in OD value of approximately $20 \%$. Analogously, the $0.05 \% \mathrm{CCPP} / \mathrm{D} / \mathrm{Col}$ scaffolds exhibited the best effect, which may be attributed to following reasons: firstly, DOPA modification successfully changed the microenvironment around the CCPP scaffolds to alkalescent, which could promote cell adhesion and proliferation. Secondly, via strong interactions between functional groups on D/CCPP and collagen, the CCPP/D/Col scaffolds obtained optimal cytocompatibility comparable to that of collagen itself. ${ }^{38,39}$ Overall, the prepared CCPP scaffolds containing low-concentration copper ions (especially $0.05 \% \mathrm{Cu}$ ) possessed good biocompatibility with no significant cytotoxicity on BMSC viability. Once further modified by collagen coating, cells seeded on them proliferated more rapidly. Therefore, the promising potential of 0.05\% CCPP/D/Col scaffolds used for bone defect restoration was preliminarily demonstrated by its compelling capacity to promote BMSCs proliferation.

\subsection{Assessment of BMSC attachment morphology and distribution}

SEM images of BMSCs were obtained after culturing them on various scaffolds for 2 days. As displayed in Fig. 5(a) and (b), there were smaller number, but well spread BMSCs attached on the initial CPP scaffolds. Whereas, conversely and unexpectedly,
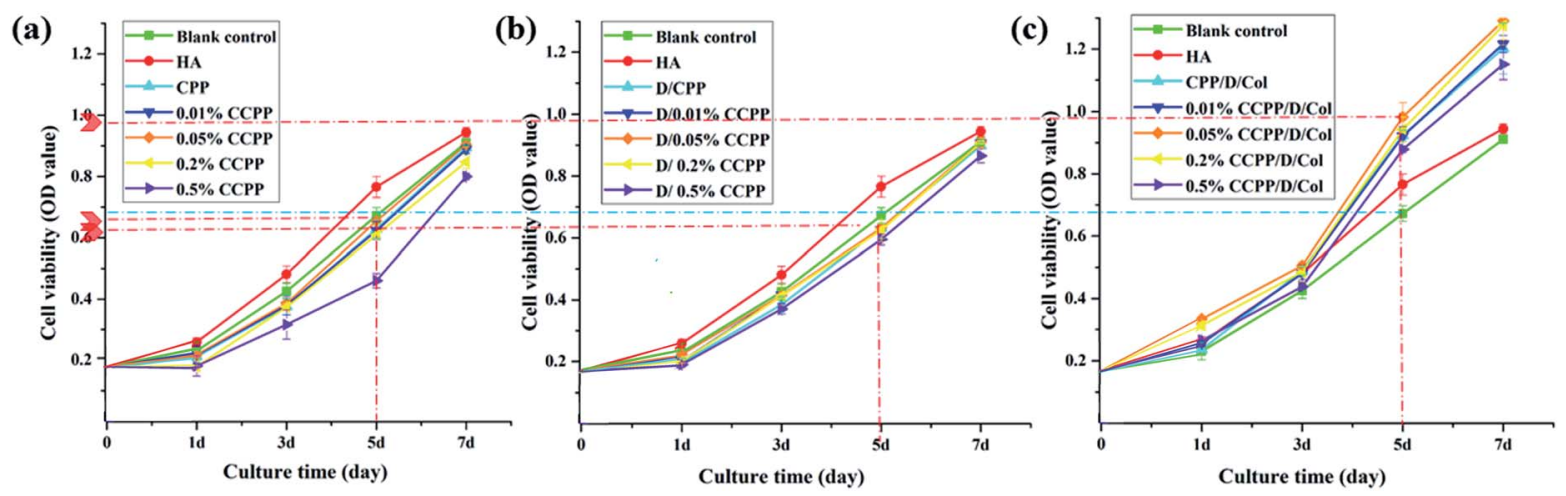

Fig. 4 Cell viability of BMSCs cultured with different scaffolds. (a) Unmodified CPP or CCPP scaffolds, (b) D/CCPP scaffolds and (c) CCPP/D/Col scaffolds. Red dotted line: compared with blank control group, the proliferation rate of BMSCs seeded on 0.05\% CCPP and its DOPA/collagen modified product on the 5th day. 

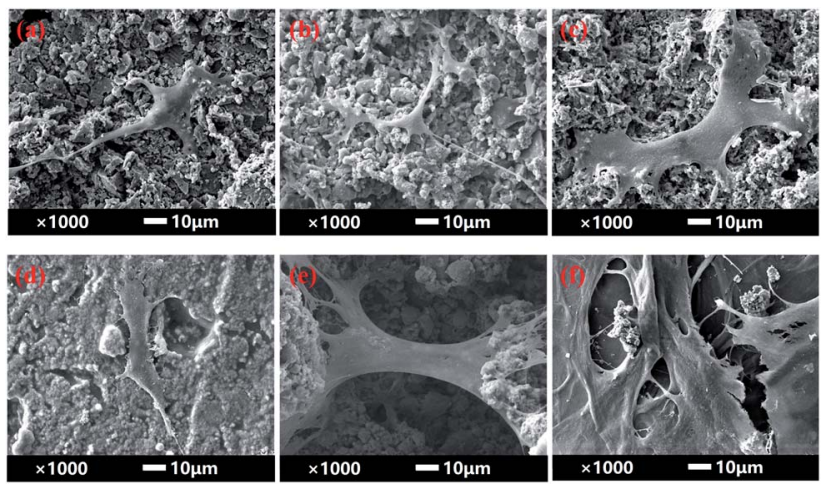

Fig. 5 The morphology of BMSCs attached on various scaffolds for 2 days. (a) CPP as control group, (b) $0.05 \%$ CCPP, (c) D/CPP, (d) D/0.05\% CCPP, (e) CPP/D/Col, and (f) 0.05\% CCPP/D/Col.

most of the BMSCs on $0.05 \%$ CCPP presented a transmutative and sparse morphology, indicating poor activity or dead cells. This was imputed to the weak-acidic situation of the local microenvironment adjacent to $0.05 \%$ CCPP, which was unbeneficial for cell growth and may accelerate the release of copper ions, causing high cytotoxicity. As shown in Fig. 5(c) and (d), after being preliminarily modified by DOPA, BMSCs on the D/ CPP and D/0.05\% CCPP scaffolds all presented well-spread morphology and lamellipodia stretched from the cell surface were evidently observed. It may be due to DOPA's modification adjusting the microenvironment to alkalescent, which could effectively promote cell proliferation and attachment. After being further modified by collagen coating, the cells became flatter and better stretched, possessing a large number of filopodia and lamellipodia adjacent to BMSCs. This result indicated that the biomimetic scaffolds prepared through further chemically coating collagen could provide an ideal environment similar to that of the ECM for BMSC growth and attachment.

Cytoskeletons were labelled and immunofluorescently stained to observe the morphology of the seeded BMSCs. As exhibited in Fig. 6, similar to the result obtained from Fig. 5(b), the globular-like BMSCs on the surface of the 0.05\% CCPP disk were simultaneously found from CLSM observation, whereas cells seeded on CPP scaffolds were smaller-numbered, but wellspread. It implied that just introducing copper ions did not benefit the spreading or proliferation of BMSCs compared with the blank control (CPP scaffolds). Merely comparing their cell number with that of the CPP sample, no obvious adverse effect was exhibited for the D/CPP group, while the amount increased significantly on the D/0.05\% CCPP one. Cells on the D/0.05\% CCPP scaffold became denser and had a criss-cross distribution. On the one hand, DOPA modification adjusted the microenvironment to alkalescent. It could guarantee the controllable release rate of copper ions and thus accelerate cell proliferation and spreading. On the other hand, the cell morphology and stretching were also affected by the microstructure of the surface. The CPP sample had a number of small-sized plate-like crystals on its surface, while the sample doped with a low concentration of $\mathrm{Cu}^{2+}$ (especially $\mathrm{D} / 0.05 \%$ CCPP), smaller and more regular crystals appeared, which were
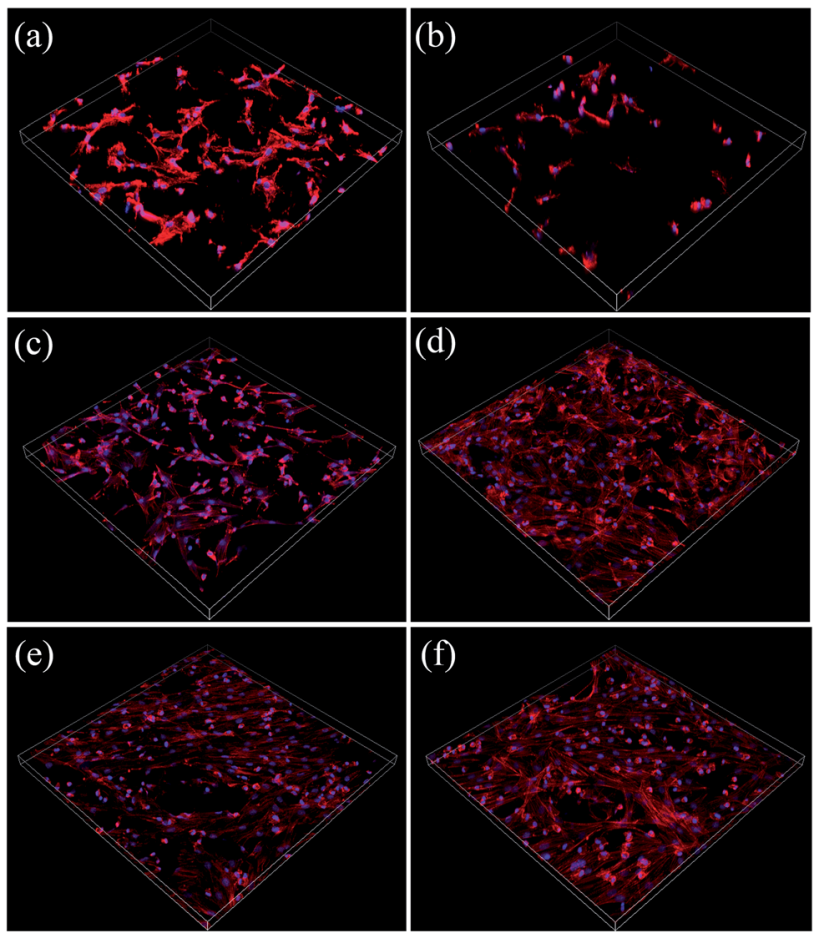

Fig. 6 Cytoskeletal organization of BMSCs grown on various scaffolds: (a) CPP, (b) 0.05\% CCPP, (c) D/CPP, (d) D/0.05\% CCPP, (e) CPP/ $\mathrm{D} / \mathrm{Col}$, and (f) $0.05 \% \mathrm{CCPP} / \mathrm{D} / \mathrm{Col}$ at the 4 th day. Cytoskeleton is stained red, while the cell nuclei are stained blue.

more suitable for cell attachment and growth. ${ }^{\mathbf{4 0 , 4 1}}$ Therefore, based on DOPA modification, $\mathrm{Cu}$ incorporation could support BMSC proliferation and attachment: cells tended to spread out extensively and even covered the porous structure of the scaffolds.

\subsection{Analysis of osteogenic differentiation}

To intuitively evaluate their osteogenic differentiation properties, ARS staining was used to detect the mineralization of the extracellular matrix after a predetermined incubation period. As displayed in Fig. 7, massive calcified nodules appeared in the
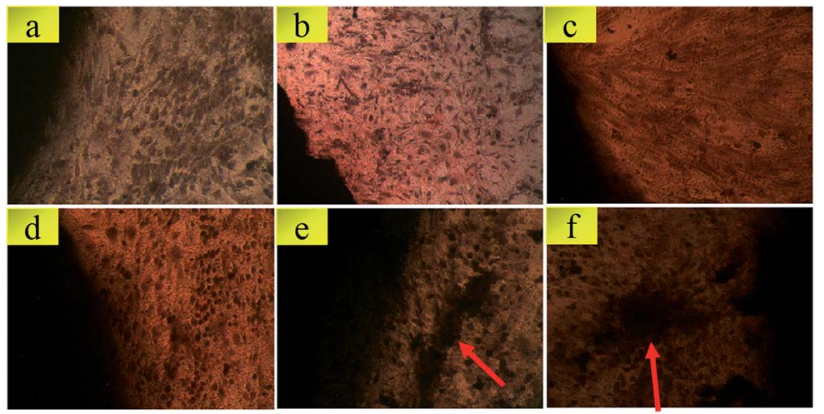

Fig. 7 Formation of extracellular matrix mineralization detected by Alizarin Red S (ARS) staining after BMSCs were seeded on various scaffolds for 28 days. The arrows indicate significant mineralized nodules. 
$\mathrm{CPP} / \mathrm{D} / \mathrm{Col}$ and $0.05 \% \mathrm{CCPP} / \mathrm{D} / \mathrm{Col}$ group; however, adjacent to the CPP and $0.05 \%$ CCPP samples, fewer nodules appeared and their Alizarin red staining was significantly lighter and smaller. As is well known, the amount of calcium nodules presented a positive relationship with the osteogenic differentiation properties of the corresponding scaffold. Since it was the highest in the $0.05 \% \mathrm{CCPP} / \mathrm{D} / \mathrm{Col}$ group and less pronounced in the initial unmodified CPP sample, it may indicate that $0.05 \%$ $\mathrm{CCPP} / \mathrm{D} / \mathrm{Col}$ scaffolds had the optimal capability to enhance osteogenic activity and promote osteogenic differentiation. Taken together, combined with the results obtained in the ARS assay and CLSM observation, in comparison with the initial CPP scaffolds, doping with moderate copper ions and further modifying through coating collagen could effectively promote BMSCs adjacent to scaffolds to form more ECM minerals and exhibit better osteogenic differentiation property.

The $0.05 \% \mathrm{CCPP} / \mathrm{D} / \mathrm{Col}$ scaffold may be a promising biomaterial for bone repair. Compared with the traditional CPP scaffold, it potentially provided an optimal environment for new bone formation, since it effectively enhanced cell-mediated osteogenesis and eliminated the brittleness.

\subsection{Angiogenesis- and osteogenesis-related gene expression}

Doping with a moderate amount of copper ions and coating with collagen may have the potential to promote new bone regeneration by enhancing osteogenesis- and angiogenesisrelated gene expression. In this research, to evaluate the role of these two modifications in osteogenic and angiogenic activity in vitro, several kinds of typical osteogenesis-(ALP, OCN, OPN) and angiogenesis-related (VEGF, HIF-1 $\alpha$ ) gene expressions were measured after BMSCs were seeded on various samples for $21 \mathrm{~d}$.

Fig. 8 shows that the incorporation of a moderate amount of copper ions (especially $0.05 \%$ ) into the initial CPP scaffold only slightly increased the expressions of these genes, whereas after secondary modification by collagen coating, their expressions increased remarkably. Therefore, as the RT-qPCR analysis displayed, the experimental group with $0.05 \%$ copper doping and collagen coating significantly up-regulated the expression of all of the investigated genes, and most of their expressions even reached a peak value at this point. In conclusion, these two-step modifications involving $\mathrm{Cu}^{2+}$ doping and collagen coating both stimulated the vascularization process, and exhibited beneficial effects on osteogenic differentiation and mineralization of BMSCs, which was in accordance with the results obtained in the ARS assay. It was attempted to explain this by the following reasons: firstly, and importantly, as previously reported, the oxygen-deficient microenvironment may be the main factor for enhancing the osteogenic differentiation of BMSCs seeded on scaffolds. $\mathrm{Cu}^{2+}$ released from the $0.05 \%$ CCPP/D/Col scaffolds could mimic a hypoxic microenvironment, in which HIF-1 $\alpha$ was stabilized by the lack of oxygen, and then further facilitate the expression of VEGF. Meanwhile, the hypoxic microenvironment also promoted osteogenic differentiation through stimulating the expression of bone-related genes, such as ALP, OCN and OPN. ${ }^{37,42,43}$ Secondly, since collagen is one of the most important components of the bone extracellular matrix, collagen coating in the secondary modification process provided more functional destinations for differentiating cells in growing bone, and thus further promoted the cells' osteogenic differentiation.

However, for the above mentioned $0.05 \% \mathrm{CCPP} / \mathrm{D} / \mathrm{Col}$ scaffolds, after further increasing the copper ion concentration (take $0.5 \% \mathrm{CCPP} / \mathrm{D} / \mathrm{Col}$ for example), almost all osteogenesisand angiogenesis-related gene expressions showed a dramatic decrease, even compared with the initial CPP scaffolds. That may because high concentrations of $\mathrm{Cu}^{2+}$ easily cause high (a)

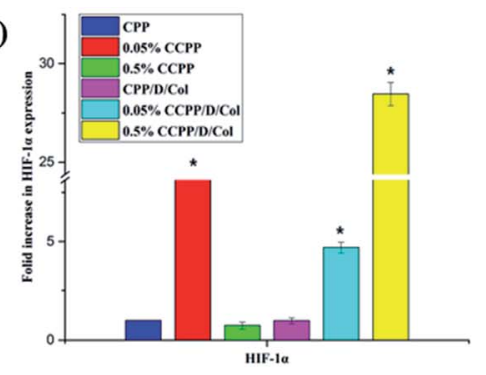

(b)

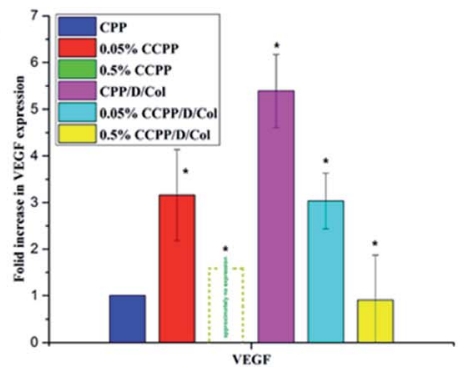

(c)

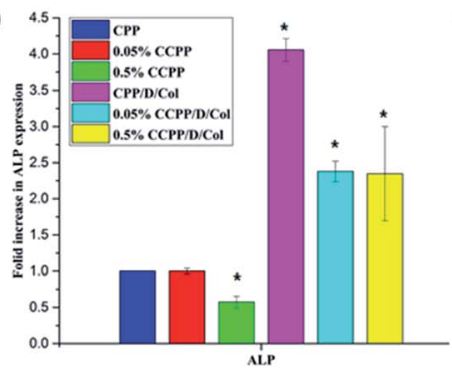

(d)

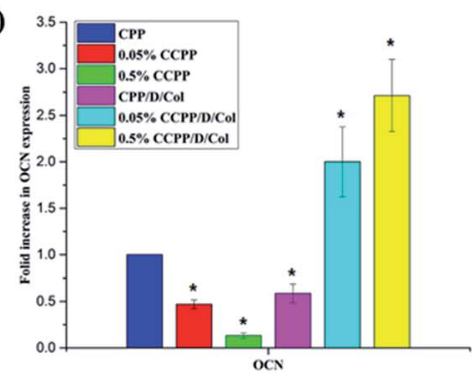

(e)

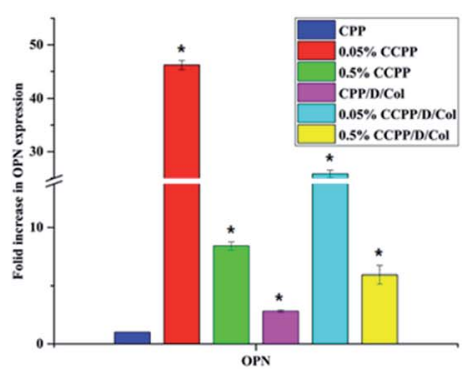

Fig. 8 Angiogenesis-related gene expression of (a) HIF-1 $\alpha$ and (b) VEGF and osteogenic-related gene expression of (c) ALP, (d) OCN, and (e) OPN of BMSCs cultured on various unmodified and collagen-modified scaffolds after 21 days. Copper doping and collagen coating significantly promoted the osteogenic differentiation of BMSCs on scaffolds. $(* p<0.05)$. 

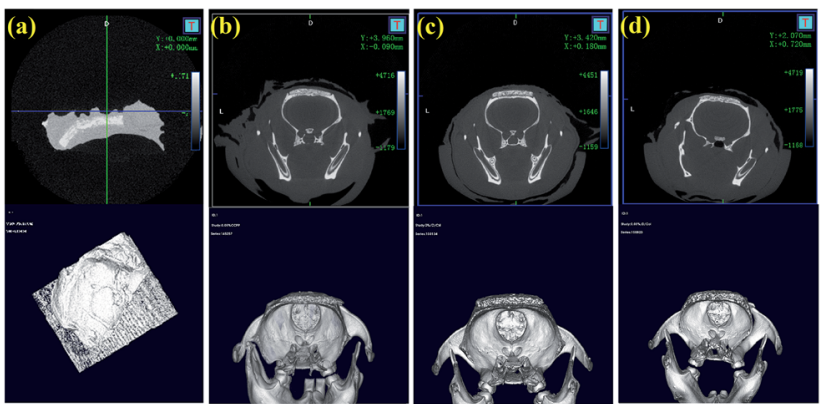

Fig. 9 Micro-CT assessment of bone reconstruction in SD rats' calvarial defects implanted with various composite scaffolds and cultivated for 8 weeks: (a) CPP, (b) $0.05 \%$ CCPP, (c) CPP/D/Col, and (d) $0.05 \%$ CCPP/D/Col.

cytotoxicity, leading to a significant inhibition of cell proliferation, differentiation and functionalization. This indicates that a low concentration of $\mathrm{Cu}^{2+}$ has a promotion effect on osteogenic and angiogenic activation, but increasing its concentration triggered an adverse effect.

\subsection{Micro-CT analysis and histological observation}

After 8 weeks of cultivation, all rats with implanted scaffolds were scanned by micro-CT and Fig. 9 exhibits the 3Dreconstructed images. As shown, with the progression of implantation time, the volumes of the scaffolds were all decreased. Furthermore, the $\mathrm{CPP} / \mathrm{D} / \mathrm{Col}$ and $0.05 \% \mathrm{CCPP} / \mathrm{D} / \mathrm{Col}$ scaffolds exhibited a more remarkable decreasing tendency compared to the others, which indicated that these two groups had better new bone formation promoting effects. They effectively induced the regeneration of bones penetrating into the porous structures of these two composite scaffolds. In addition, good fusion between the newly generated mineralized layers and host bones is critical for bioactive scaffolds to rapidly bond to living bone in vivo. As displayed, an obvious gap was situated at the periphery of the unmodified CCPP implants and old bones, while no gaps were visible once they were further modified by collagen coating. This indicated that the composite scaffolds, obtained from secondary collagen modification, had a more prominent ability to integrate with host bones and fused with them more easily.

\section{Conclusions}

In this study, via introducing DOPA to form stable bonds, we successfully prepared $0.05 \% \mathrm{CCPP} / \mathrm{D} / \mathrm{Col}$ to obtain multifunctional composite scaffolds fit for bone regeneration and reconstruction. As confirmed, without cell proliferation, the controllable copper ion release and collagen stripping could effectively induce the osteogenesis differentiation of BMSCs through activating bone-related genes and stimulating protein expression, further accelerating vascularization and new bone formation. In addition, collagen coating endowed the initial CPP scaffolds with excellent toughness and compressive strength. In a word, CCPP/D/Col scaffolds will evolve as promising multifunctional biomaterials for bone reconstruction. The concept, introducing bioactive $\mathrm{Cu}$ ions and collagen into inert CPP scaffolds to develop an innovative biocompatible and bioresorbable composite scaffold with excellent angiogenesis and osteogenesis properties, may offer a new method to fabricate multifunctional composite scaffolds for bone regeneration.

\section{Conflicts of interest}

There are no conflicts to declare.

\section{Acknowledgements}

The authors would like to thank the National Key Research and Development Program of China (No. 2016YFC1100900, No. 2016YFC1100901, No. 2016YFC1100903, No. 2016YFC1100904), the National Nature Science Foundation of China (No. 81401528) and the Scientific and Technological project of Chengdu (No. 2014-HM01-00207-SF) and Sichuan UniversityLuzhou Strategic Partnership Scientific and Technological Cooperation Projects (No. 2014CDLZ-S27).

\section{Notes and references}

1 T. Genova, L. Munaron, S. Carossa and F. Mussano, J. Biomater. Appl., 2016, 30, 940-951.

2 R. A. Carano and E. H. Filvaroff, Drug Discovery Today, 2003, 8, 980-989.

3 R. Pilliar, M. Filiaggi, J. Wells, M. Grynpas and R. Kandel, Biomaterials, 2001, 22, 963-972.

4 G. Caetano, R. Violante, A. B. Sant, A. B. Murashima, M. Domingos, A. Gibson, P. Bártolo and M. A. Frade, Mater. Lett., 2016, 182, 318-322.

5 C. You, M.-H. Lee, H.-J. Lee, M.-H. Han, T.-Y. Kwon, K.-H. Kim and D. S. Oh, Ceram. Int., 2017, 43, 3540-3546.

6 F. M. Wang, K. Qiu, T. Hu, C. X. Wan, X. D. Zhou and J. Gutmann, Int. Endontic. J., 2006, 39, 477-483.

7 J. T. Ratnayake, M. L. Gould, A. Shavandi, M. Mucalo and G. J. Dias, J. Biomed. Mater. Res., Part B, 2017, 105, 1054-1062.

8 D. M. Vasconcelos, S. G. Santos, M. Lamghari and M. A. Barbosa, Biomaterials, 2016, 84, 262-275.

9 S. Bose, G. Fielding, S. Tarafder and A. Bandyopadhyay, Trends Biotechnol., 2013, 31, 594-605.

10 X. Wang, Z. Gu, B. Jiang, L. Li and X. Yu, Biomater. Sci., 2016, 4, 678-688.

11 S. Stegen, S. Deprez, G. Eelen, S. Torrekens, R. Van Looveren, J. Goveia, B. Ghesquière, P. Carmeliet and G. Carmeliet, Bone, 2016, 87, 176-186.

12 Q. Li, H. Chen, X. Huang and M. Costa, Toxicol. Appl. Pharmacol., 2006, 213, 245-255.

13 S. B. Devi and K. Sulochana, J. Biosci., 2016, 41, 487-496.

14 H. Wang, S. Zhao, J. Zhou, Y. Shen, W. Huang, C. Zhang, M. N. Rahaman and D. Wang, J. Mater. Chem. B, 2014, 2, 8547-8557.

15 I. Burghardt, F. Lüthen, C. Prinz, B. Kreikemeyer, C. Zietz, H.-G. Neumann and J. Rychly, Biomaterials, 2015, 44, 36-44.

16 D. R. Eyre and M. A. Weis, Calcif. Tissue Int., 2013, 93, 338347. 
17 K. Jiao, L.-n. Niu, Q.-h. Li, F.-m. Chen, W. Zhao, J.-j. Li, J.-h. Chen, C. W. Cutler, D. H. Pashley and F. R. Tay, Acta Biomater., 2015, 19, 23-32.

18 Y. Wen, S. L. Roudebush, G. A. Buckholtz, T. R. Goehring, N. Giannoukakis, E. S. Gawalt and W. S. Meng, Biomaterials, 2014, 35, 5196-5205.

19 Y. Hu, W. Dan, S. Xiong, Y. Kang, A. Dhinakar, J. Wu and Z. Gu, Acta Biomater., 2017, 47, 135-148.

20 R. Sa, Y. Yan, Z. Wei, L. Zhang, W. Wang and M. Tian, ACS Appl. Mater. Interfaces, 2014, 6, 21730-21738.

21 J. Zhao, Z. Li, M. Zhang, A. Meng and Q. Li, ACS Sustainable Chem. Eng., 2016, 4, 3598-3608.

22 J.-H. Jiang, L.-P. Zhu, X.-L. Li, Y.-Y. Xu and B.-K. Zhu, J. Membr. Sci., 2010, 364, 194-202.

23 G. Sun, S. Kusuma and S. Gerecht, Macromol. Biosci., 2012, 12, 21-28.

24 M. Shi, Y. Zhou, J. Shao, Z. Chen, B. Song, J. Chang, C. Wu and Y. Xiao, Acta Biomater., 2015, 21, 178-189.

25 S. Y. Hou, H. Y. Zhang, D. P. Quan, X. L. Liu and J. K. Zhu, J. Neurosci., 2006, 140, 101-110.

26 K. Qiu, X. J. Zhao, C. X. Wan, C. S. Zhao and Y. W. Chen, Biomaterials, 2006, 27, 1277-1286.

27 L. Zhang, J. Shi, Z. Jiang, Y. Jiang, S. Qiao, J. Li, R. Wang, R. Meng, Y. Zhu and Y. Zheng, Green Chem., 2011, 13, 300306.

28 K. Hayashi, T. Inadome, T. Mashima and Y. Sugioka, J. Biomed. Mater. Res., 1993, 27, 557-563.

29 S. R. B. Devi, A. M. Dhivya and K. N. Sulochana, J. Biosci., 2016, 41, 487-496.

30 J. P. Rodriguez, S. Rios and M. Gonzalez, J. Cell. Biochem., 2002, 85, 92-100.
31 L. Yu, G. Jin, L. Ouyang, D. Wang, Y. Qiao and X. Liu, J. Mater. Chem. B, 2016, 4, 1296-1309.

32 W. Hu, S. Lu, Y. Ma, P. Ren, X. Ma, N. Zhou, T. Zhang and Z. Ji, J. Mater. Chem. B, 2017, 5, 575-585.

33 M. Dressler, F. Dombrowski, U. Simon, J. Börnstein, V. D. Hodoroaba, M. Feigl, S. Grunow, R. Gildenhaar and M. Neumann, J. Eur. Ceram. Soc., 2011, 31, 523-529.

34 E. Sharifi, M. Azami, A. M. Kajbafzadeh, F. Moztarzadeh, R. Faridi-Majidi, A. Shamousi, R. Karimi and J. Ai, Mater. Sci. Eng., C, 2016, 59, 533-541.

35 H. Lee, S. M. Dellatore, W. M. Miller and P. B. Messersmith, Science, 2007, 318, 426-430.

36 X. Li, M. Fu, J. Wu, C. Zhang, X. Deng, A. Dhinakar, W. Huang, H. Qian and L. Ge, Acta Biomater., 2017, 51, 294-303.

37 Q. Wu, J. Li, W. Zhang, H. Qian, W. She, H. Pan, J. Wen, X. Zhang, X. Liu and X. Jiang, J. Mater. Chem. B, 2014, 2, 6738-6748.

38 G. Sun, Y. I. Shen, C. C. Ho, S. Kusuma and S. Gerecht, J. Biomed. Mater. Res., 2010, 93, 1080-1090.

39 Y. Wen, W. Liu, C. Bagia, S. Zhang, M. Bai, J. M. Janjic, N. Giannoukakis, E. S. Gawalt and W. S. Meng, Acta Biomater., 2014, 10, 4759-4767.

40 G. Sun, Y. I. Shen, S. Kusuma, K. Fox-Talbot, C. J. Steenbergen and S. Gerecht, Biomaterials, 2011, 32, 95-106.

41 C. M. Murphy, M. G. Haugh and F. J. O'Brien, Biomaterials, 2010, 31, 461-466.

42 W. Zhang, Q. Chang, L. Xu, G. Li, G. Yang, X. Ding, X. Wang, D. Cui and X. Jiang, Adv. Healthcare Mater., 2016, 5, 1247.

43 C. Wu, Y. Zhou, J. Chang and Y. Xiao, Acta Biomater., 2013, 9, 9159-9168. 